\title{
Doppelschlag gegen HER2 verspricht Erfolg
}

Die Kombination der beiden monoklonalen HER2-Antikörper Trastuzumab und Pertuzumab verlängert das progressionsfreie Überleben von Patientinnen mit metastasiertem HER2-positivem Mammakarzinom, so die Ergebnisse einer aktuellen Studie. Eine übermäßige Zunahme kardiotoxischer Effekte steht dabei offenbar nicht zu befürchten.

$\mathrm{n}$ der CLEOPATRA-Studie (Clinical

Evaluation of Pertuzumab and Trastuzumab) erhielten 402 Patientinnen mit einem lokalen, nicht operablen Rezidiv bzw. Metastasen eines HER2-positiven Mammakarzinoms, Trastuzumab und Pertuzumab neben mindestens sechs $\mathrm{Zy}$ klen Docetaxel. Beide HER2-Antikörper wurden als dreiwöchentliche Dauertherapie bis zur Progression der Erkrankung bzw. bis zum Auftreten intolerabler Nebenwirkungen verabreicht. Die 406 Patientinnen der Kontrollgruppe wurden auch mit Docetaxel und Trastuzumab behandelt, statt Pertuzumab bekamen sie Placebo. Primärer Studienendpunkt war das progressionsfreie Überleben (PFS).

In der Kontrollgruppe betrug das PFS im Median 12,4 Monate, in der Pertuzumab-Gruppe 18,5 Monate - das Progressionsrisiko sank um 38\%. Für die Gesamtüberlebensraten (overall survival, OS), einen sekundären Endpunkt von CLEOPATRA, war zwar nach gut 19-monatigem Follow-up ein Trend zugunsten der Kombination von Trastuzumab plus Pertuzumab abzulesen ( $82,8 \%$ vs. $76,4 \%$ ). Der Unterschied war aber nicht signifikant (es war eine Interimsanalyse; belastbare OS-Daten werden Ende 2013 erwartet.

Im Sicherheitsprofil schnitt die Kombination etwas schlechter ab, z.B. gab es unter Pertuzumab mehr Diarrhöen, $\mathrm{Mu}$ kositiden und febrile Neutropenien, auch Nebenwirkungen vom Grad 3 oder höher traten häufiger auf. Bei 8,3\% der Patientinnen in der Pertuzumab- und bei 4,4\% in der Kontrollgruppe kam es zu linksventrikulärer systolischer Dysfunktion, bei 2,8\% bzw. 1,2\% war der Schweregrad 3 oder höher.

Fazit: Pertuzumab und Trastuzumab greifen an verschiedenen Rezeptorepitopen an und wirken komplementär - ihre Kombination könnte sich also auszahlen. Gegenüber Trastuzumab plus Placebo ver- längerte die Kombination mit Pertuzumab das PFS der HER2-positiven Brustkrebspatientinnen. Ob sich der günstige Effekt auch in einem signifikant höheren OS niederschlägt, bleibt abzuwarten.

Baselga J et al. Pertuzumab plus Trastuzumab plus Docetaxel for Metastatic Breast Cancer. N Engl J Med 2012; 366: 109-19

Kommentar: Im Dezember 2011 präsentierte Baselga erstmals die CLEOPATRAErgebnisse in San Antonio. Lange hatte man dort auf signifkante Ergebnisse einer Phase-III-Studie in der metastasierten Situation beim Mammakarzinom gewartet. Zeitgleich zum Kongress erschien diese Publikation im New England Journal of Medicine.

Pertuzumab zeigt in dieser Phase-III-Studie von 2008-2010 einen ergänzenden Effekt zur Trastuzumab-Monotherapie in der typischen Kombination mit Docetaxel in der First-Line-Behandlung des metastasierten HER2-positiven Mammkarzinoms.

Für die Studie erfolgte eine Qualitätssicherung der Pathologie durch zentrale Re-Testung des HER2-Status, sicher eine Stärke dieses Studienprotokolls, ebenso die Mindestdauer von sechs Therapiezyklen für die Auswertung. In die Studie wurden sowohl Frauen mit viszeraler wie nichtviszeraler Metastasenlokalisationen aufgenommen mit einer Gleichverteilung des Hormonrezeptorstatus sowie mit einer vorherigen (neo)adjuvanten Therapie (inklusive TrastuzumabVorbehandlung). Kritisch bewertet werden muss die Tatsache, dass Hirnmetastasen von der Randomisierung ausgeschlossen waren, sie aber gerade bei HER2-positiven Patientinnen häufig vorliegen. Hier bleiben entsprechende weitere Studien abzuwarten. Das primäre Studienendziel, die Verbesserung des progressionsfreien Überlebens, konnte signifikant verlängert werden. In der Subgruppenanalyse konnte dieser signifikante Effekt ebenfalls in allen Parametern bestätigt werden, auch nach Trastuzumab-Vorbehand- lung (neo)adjuvant (PFS: 16,9 vs. 10,4 Monate) bzw. mit (neo)adjuvanter Chemotherapievorbehandlung (PFS: 21,6 vs. 12,6 Monate), ebenso unabhängig von Alter, Region, ethnischer Zugehörigkeit, Metastasenlokalisation, Hormonrezeptorstatus und HER2Score-Positivität. Die objektive Ansprechrate liegt bei $80,2 \%$ versus $69,3 \%$ : ein Vorteil von über $10 \%$ für den Behandlungsarm bei dieser prognostisch ungünstigen Situation. Bezüglich des Gesamtüberlebens liegt noch keine Endauswertung vor (derzeitiges Follow-up 19,3 Monate), ein starker Trend zugunsten der Pertuzumab-Kombination zeichnet sich ab $(H R<0,603)$. Bezüglich der Sicherheitsdatenanalyse konnte gezeigt werden, dass keine zusätzliche kardiotoxe Wirkung in der Kombination von Trastuzumab plus Pertuzumab zu erwarten ist, wenn bei Medikationsbeginn eine LVEF > 50\% vorliegt; die sonstigen Grad-III- und -IV-Nebenwirkungen waren besonders die afebrile Neutropenie $(13,8 \%$ vs. $7,6 \%)$ und Diarrhoe $(66,8 \%$ vs. 46,3\%) - beides in den Händen mit neuen Substanzen versierter Mamma-Onkologen beherrschbare Toxizitäten.

Der Einsatz von Trastuzumab sowohl beim primären als auch beim metastasierten Mammakarzinom war der Durchbruch für die ca. 20\% der HER2-positiven prognostisch ungünstigen Mammkarzinompatientinnen. Bei der Suche nach synergistischen Effekten in der (vollständigen) Blockade der HER2-Kaskade wird nun mit dem Kombinationspartner Pertuzumab ein relevanter klinischer Erfolg durch die Bindung an andere Epitope des HER2-Rezeptors und damit Einfluss auf die Rezeptordimerisierung gezeigt. Das Konzept der dualen Blockade, d.h. der komplementären Kombination von HER2-aktiven Substanzen in der individualisierten Therapie, wird mit der CLEOPATRA-Studie bestätigt.

Die positiven Ergebnisse von Pertuzumab werden ergänzt durch erste Erfahrungen mit der Substanz in der neoadjuvanten Therapie, wie in Neosphere oder auch beim SABCS 2011 gezeigt: In der TRYPHAENEA-Studie wurden pCR-Raten von über $50 \%$ erreicht.

In Deutschland ist die Substanz bisher nicht zugelassen - auf Basis der CLEOPATRADaten ist die Zulassung jedoch eingereicht.

Wir werden im Rahmen der APHINITYStudie in vielen Studienzentren in Deutschland die Gelegenheit haben, Erfahrung mit der Substanz in der adjuvanten Therapie des HER2-positiven Mammkarzinoms zu sammeln. 